\title{
DISTRIBUIÇÃO ESPAÇO-TEMPORAL DE Batillipes pennaki MARCUS, 1946 (TARDIGRADA, HETEROTARDIGRADA) NA MARGEM SUL DA ILHA DE ITAMARACÁ (PERNAMBUCO - BRASIL).
}

Clélia Márcia Cavalcanti da ROCHA

Depto. de Biologia - UFRPE

Depto. Oceanografia - UFPE ( PPGO)

Verônica FONSÊCA-GENEVOIS

Depto. de Zoologia - UFPE

Francisco José Victor CASTRO

Depto. de Oceanografia ( PPGO) - UFPE

\section{RESUMO}

Populações de Batillipes pennaki Marcus, 1946 integrantes da meiofauna de 3 estações em zona intermareal da margem Sul da ilha de Itamaracá, litoral norte de Pernambuco, localizadas entre $34^{\circ}$ e $34^{\circ} 52^{\prime} \mathrm{W}$ e $7^{0} 49^{\prime} \mathrm{S}$ foram prospectadas, a fim de se estimar suas variações espaço-temporais . O material bio-sedimentológico foi coletado por um ano a cada 3 meses durante as preamares e baixa-mares.Valor máximo de densidade ocorreu no médio-litoral superior arenoso durante a preamar. Detectou-se ainda um gradiente de densidade populacional negativo no sentido continente-mar.

Palavras - chave : Meiofauna, Tardigrada, Batillipes pennaki, Zona intermareal,

\section{ABSTRACT \\ Spatio-Temporal Distribution of Batillipes pennaki Marcus, 1946 at the Southern of Itamaraca Island (Pernambuco - Brasil) .}

Populations of Batillipes pennaki Marcus, 1946 ocurring at three intertidal stations at southern of the Itamaracá island $\left(34^{0} 50^{\prime}-34^{0} 52^{\prime} \mathrm{W}\right.$ and $\left.7^{0} 49^{\prime} \mathrm{S}\right)$ were prospected to point out their spatio-temporal distribution. Biosedimentologic samples were collected every 3 months during a year at two major phases of the tidal cycle. They showed density pics at the upper mesolittoral in january although still numerous in the lower mesolittoral, while they were almost absent in the infralittoral.

Key-words: Meiofauna, Tardigrada, Batillipes pennaki, Intertidal zone.

\section{INTRODUÇÃO}

Apesar do interesse de que se revestem os Tardigrada em relação às comunidades meiofaunísticas de que fazem parte, estudos aprofundados sobre suas populações só vieram a ser mais extensamente desenvolvidos a partir de 1928, com o advento dos trabalhos monográficos de Ernest Marcus (Higgins, 1974). A razão para isso teria sido, segundo Renaud-Mornant e Pollock (1971) a dificuldade de amostragem, extração, o diminuto tamanho desses animais e a extrema dificuldade para se distinguir-lhes o sexo, principalmente em caso de indivíduos imaturos.

A distribuição geográfica dos Tardigrada em muitos casos não está bem definida, mas, segundo Grimaldi De Zio et. al. (1983), em areias intermareais são 
encontradas essencialmente as espécies endopsâmicas, e principalmente as da família Batillipedidae. De uma forma geral pode-se acrescentar, de acordo com RenaudMornant e Pollock (op. cit.), que o gênero Batillipes spp., referido no presente trabalho, tem distribuição cosmopolita, uma vez que já foi citado para os dois Hemisférios, nos oceanos Atlântico, Pacífico, Indo-Pacífico e Mar Mediterrâneo.

A meiofauna da ilha de Itamaracá e do Canal de Santa Cuz é ainda pouco conhecida do ponto de vista da sistemática dos seus taxa, tendo sido os Copepoda Harpacticoida apontados por Wandeness et al. ( 1998) em sedimentos arenosos e por Gomes ( 1999) em sedimentos areno-lamosos. O presente trabalho objetivou fazer um estudo sistemático e biológico da população de Tardigrada na zona entre-marés da margem Sul da referida ilha, analisando flutuações espaço-temporais desta fauna ao longo de um ciclo anual.

\section{MATERIAL E MÉTODOS}

Este estudo foi efetuado na margem Sul da ilha de Itamaracá, voltada para o Canal de Santa Cruz, entre as localidades de Orange e Vila Velha, compreendendo a área delimitada pelos meridianos $34^{\circ} 50^{\prime}$ e $34^{0} 52^{\prime}$ Oeste, à altura do paralelo $7^{0} 49^{\prime}$ Sul (Fig. 1). Foram estabelecidas 3 estações de coleta, amostradas trimestralmente durante um ciclo anual, no médio- litoral superior e inferior e no infralitoral, durante os períodos de baixa e preamar. Para se definir os andares bênticos, utilizou-se o conceito de Rützler (1969). O cálculo da altura de maré por ocasião das coletas foi feito tomando-se por base as Tábuas de Marés (Ministério da Marinha - DHN) para o porto do Recife.

Amostras bio-sedimentológicas foram coletadas através de um testemunhador cilíndrico de PVC com área interna de $10 \mathrm{~cm}^{2}$, nos moldes daquele descrito por Hope (In: Hullings e Gray, 1971). Em cada ponto foram colhidas 3 réplicas, delimitadas verticalmente pelo início da camada sedimentar redutora. Fixou-se o material coletado com formol salino a $4 \%$.

Em laboratório, utilizou-se rotineiramente a metodologia proposta por Elmgren (1966) para extração da meiofauna, fazendo-se uso de peneiras geológicas com aberturas de malha de 1,00 e $0,044 \mathrm{~mm}$, e placa de Dolfuss para triagem sob lupa binocular. Todos os componentes da meiofauna foram extraídos e agrupados a níveis taxonômicos altos, sendo expressa a densidade de cada táxon em números absolutos por área de $10 \mathrm{~cm}^{2}$ de sedimento, após estimada a média das réplicas. Os Tardigrada tiveram seu comprimento corpóreo medido através de observacão direta com lente ocular micrométrica, segundo procedimento de Renaud-Debyser e Salvat (1963), para determinacão da faixa etária. Para o estudo taxonômico e biológico, confeccionou-se lâminas permanentes para microscopia, segundo técnica de Renaud-Debyser e Salvat (op. cit.).

Quatro questões básicas foram investigadas estatisticamente: utilizou-se o teste " $\mathrm{T}$ " de Wilcoxon para responder se a densidade média da meiofauna total era significativamente afetada por fases de marés; o teste "U" de Mann- Whitney para avaliar a influência do efeito temporal e do nível topográfico sobre as populações abordadas; e o teste de Kruskall - Wallis para definir o significado das diferenças encontradas entre as populações dos três transects. 


\section{RESULTADOS}

Os Tardigrada, pertencentes na sua totalidade à espécie Batillipes pennaki Marcus, 1946, ocorreram em 59\% das amostras analisadas, estando os adultos do sexo masculino presentes em $45,8 \%$ e os do sexo feminino em $48,6 \%$ do total de amostras. Os indivíduos jovens, menores que $0,2 \mathrm{~mm}$ (dimensão esta que impossibilita a distinção dos sexos) ocorreram em $38,8 \%$ das amostras estudadas.

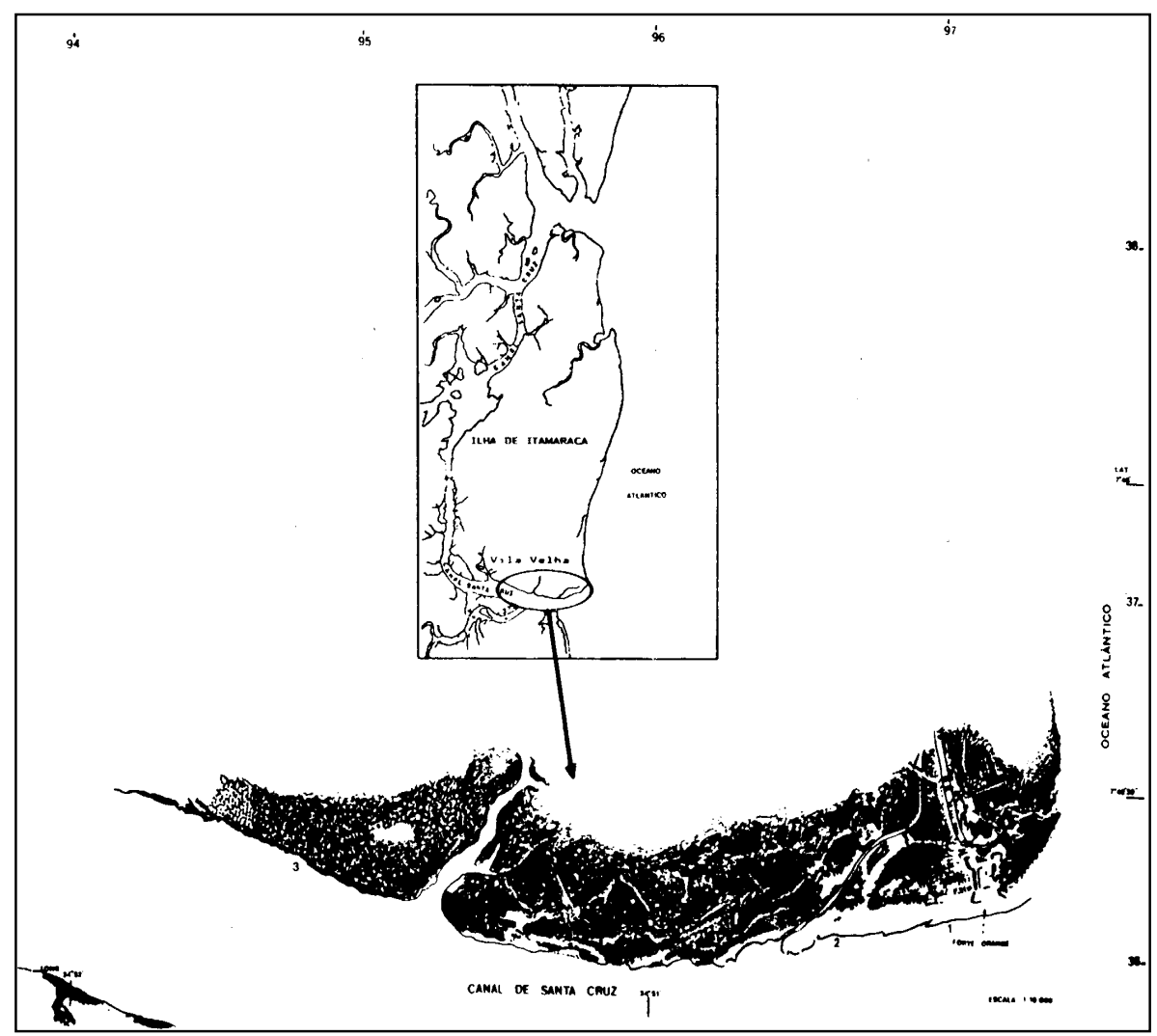

Fig. 1 - Mapa da área estudada, com localização das estações (1= estação $1 ; 2=$ estação 2; 3= estação 3) - baseado na ortofotocarta A295,9135 FIDEM - PE.

\section{Distribuição Espacial}

$\mathrm{Na}$ estação 1 (Forte Orange), composta por areia média, Batillipes pennaki esteve representado no médio-litoral inferior em sua densidade máxima para todo o 
período de estudo, com 1.300 ind. $10^{-2} \mathrm{~cm}$ durante a maré alta, sendo 575 adultos do sexo feminino, 245 do sexo masculino e 480 jovens (Figs. 2 e 7). Nos demais andares bênticos a espécie ocorreu em densidades inferiores, sendo registradas densidades de 314 ind. $10 \mathrm{~cm}^{-2}$ no médio-litoral superior na preamar e 130 ind. $10 \mathrm{~cm}^{-2}$ no infralitoral no mesmo regime de maré ( Figs 2, 5 e 8 ).

Na estação 2 (Vila Velha), composta por areia média, B. pennaki apresentou baixas densidades populacionais durante todo o ciclo anual estudado, tendo, inclusive, estado praticamente ausente no infra e médio-litoral inferior (Fig. 2). A densidade máxima,de 56 ind. $10 \mathrm{~cm}^{2}$, todos jovens, foi detectada no médio-litoral superior durante a baixa-mar.( Figs. 2 e 3 ).

$\mathrm{Na}$ estação 3 (Pedra da Macaibeira),com sedimentos areno-lamosos, os Tardigrada apresentaram densidades populacionais mais expressivas que aquelas observadas na estação 2 , mas, à semelhança desta última, praticamente não ocorreram no infralitoral. Os Tardigrada apresentaram pico de 134 ind. $10 \mathrm{~cm}^{-2}$ no médio-litoral superior durante a baixa-mar, sendo 19 jovens, 77 adultos do sexo masculino e 38 do sexo feminino (Figs. 2 e 3 ).

Para responder se as três estações eram diferentes entre si aplicou-se o teste de Kruskal-Wallis, obtendo-se resultados significativos entre elas. Com relação ao significado da variação do nível topográfico sobre a população estudada utilizou-se o teste "U" de Mann-Whitney, que indicou serem significativas as diferenças observadas entre os três níveis abordados do litoral.

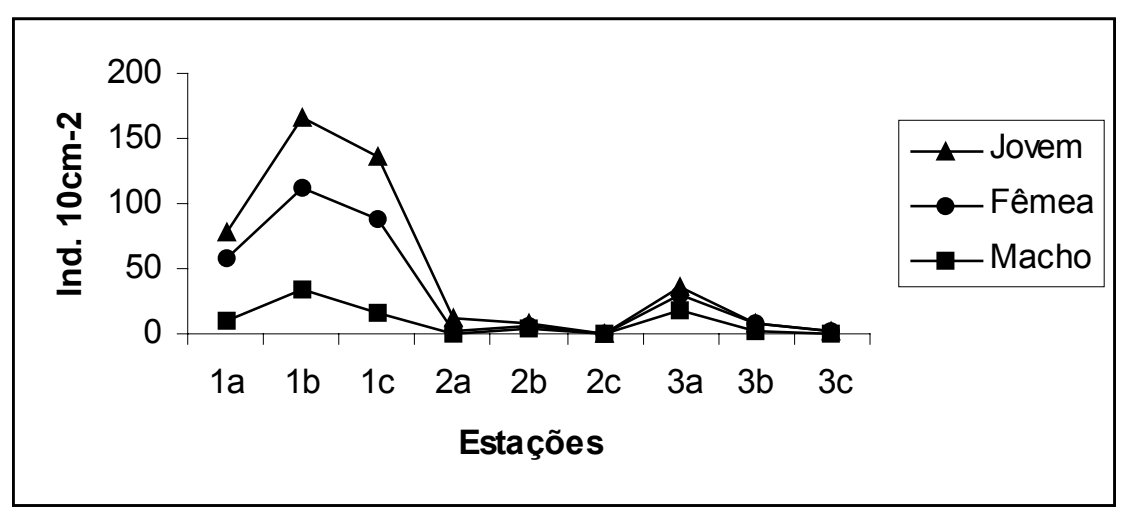

Figura 2 - Distribuição espacial da densidade média dos Tardigrada na Ilha de Itamaracá- $\mathrm{PE} \quad(\mathrm{a}=$ =médio-litoral superior, $\mathrm{b}=$ =médio-litoral inferior, $\mathrm{c}=$ infralitoral).

\section{Distribuição Temporal}

Para testar se as densidades médias da meiofauna eram semelhantes no sentido temporal utilizou-se o teste "U" de Mann-Whitney . Comparados os quatro períodos de coleta em cada estação, a hipótese Ho foi aceita na estação II, indicando não haver 38 
diferença significativa nas variações temporais de densidade. A hipótese foi rejeitada entre as estações I e III atestando que estas eram significativamente diferentes ao longo do ciclo anual.

Testadas as diferenças entre as densidades médias da meiofauna total entre baixa mar e preamar, a hipótese nula foi aceita através do teste"T" de Wilcoxon, salvo para o infralitoral da estação II. No entanto, aplicando-se o mesmo teste para as densidades do grupo aqui destacado houve diferença significativa entre as fases de maré na estação I.

As densidades máximas dos Tardigrada ocorreram durante as preamares no três andares bênticos(Figs. 4, 6 e 8). O médio-litoral superior apresentou populações compostas por machos, fêmeas e jovens nas baixamares do ciclo anual prospectado, salvo em julho, quando só jovens integraram a população. Populações completas ( machos, fêmeas e jovens) ocorreram no mês de janeiro, independente dos fatores andar bêntico e regime de marés (Figs.3 a 8 ).

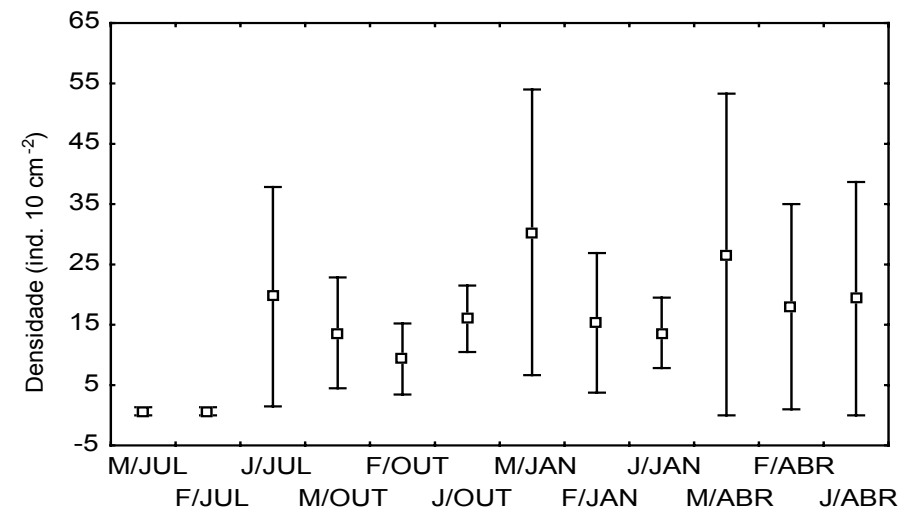

Figura 3 - Variação da densidade média e erro padrão dos Tardigrada no médio-litoral superior durante a baixa-mar na Ilha de Itamaracá, PE $(\mathrm{M}=$ machos; $\mathrm{F}=$ fêmeas; J=jovens; Jul=julho, Out=outubro, Jan=janeiro, Abr=abril). 


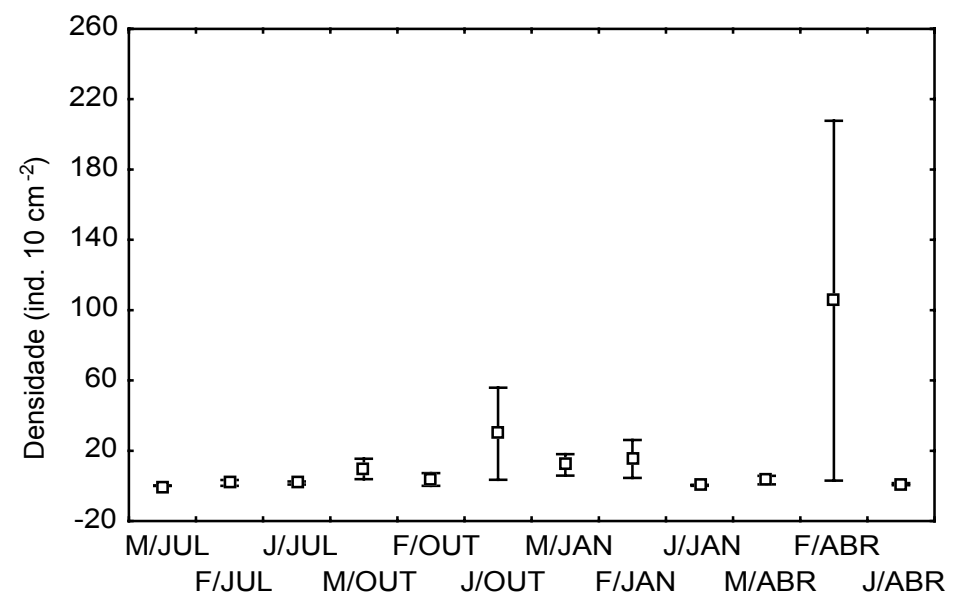

Figura 4 - Variação da densidade média e erro padrão dos Tardigrada no médio-litoral superior durante a preamar na Ilha de Itamaracá, PE. (M=machos; $\mathrm{F}=$ fêmeas; J=jovens; Jul=julho, Out=outubro, Jan=janeiro, Abr=abril).

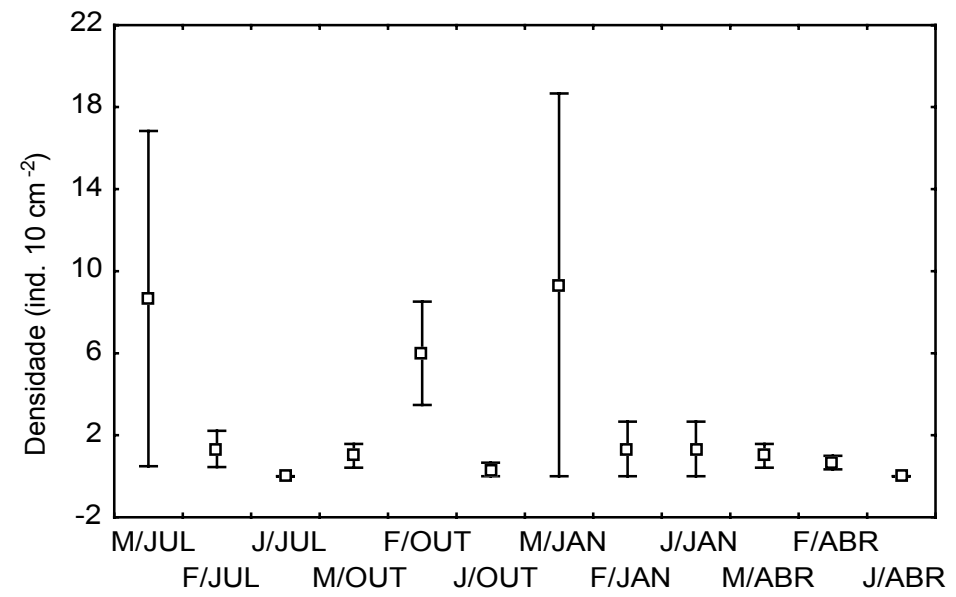

Figura 5 - Variação da densidade média e erro padrão dos Tardigrada no médio-litoral inferior durante a baixa-mar Ilha de Itamaracá, PE ( $\mathrm{M}=$ machos; $\mathrm{F}=$ fêmeas; J=jovens; Jul=julho, Out=outubro, Jan=janeiro, Abr=abril). 


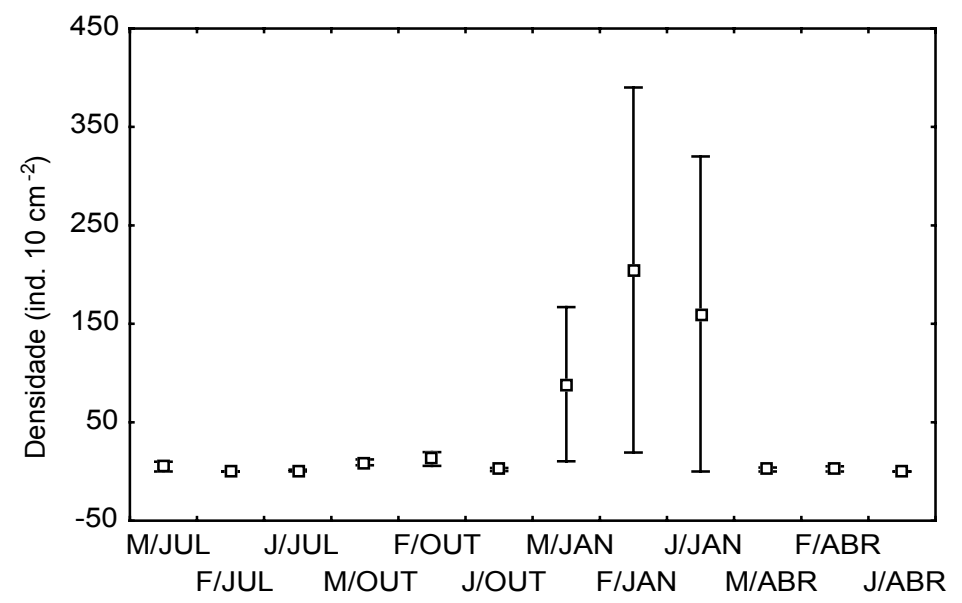

Figura 6 - Variação da densidade média e erro padrão dos Tardigrada no médio-litoral inferior durante a preamar na Ilha de Itamaracá, $\mathrm{PE}(\mathrm{M}=$ machos; F=fêmeas; $\mathrm{J}=$ jovens; Jul=julho, Out=outubro, Jan=janeiro, Abr=abril).

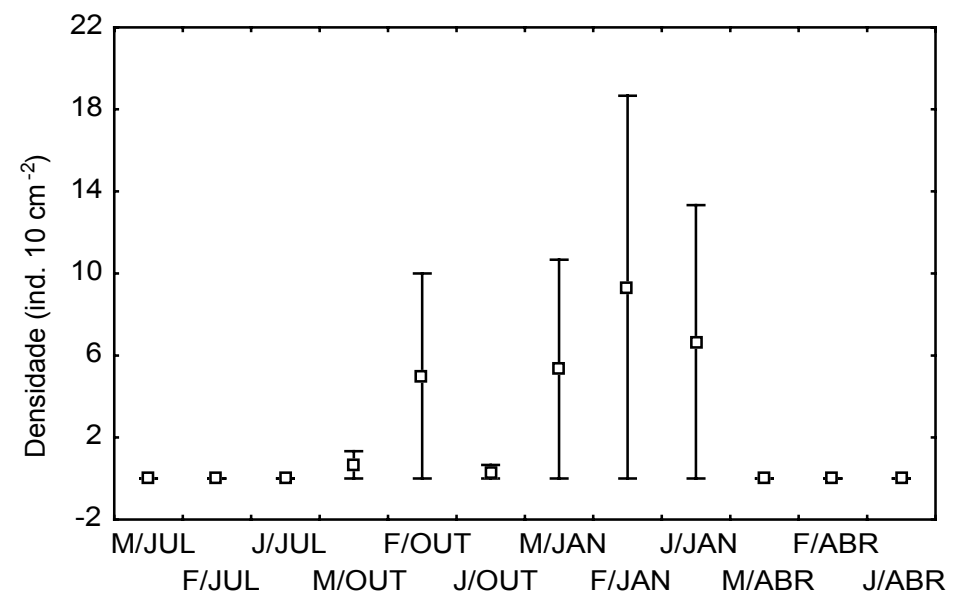

Figura 7 - Variação da densidade média e erro padrão dos Tardigrada no infralitoral durante a baixa-mar na Ilha de Itamaracá, $\mathrm{PE}(\mathrm{M}=$ machos; $\mathrm{F}=$ =ềmeas; $\mathrm{J}=$ jovens; Jul=julho, Out=outubro, Jan=janeiro, Abr=abril). 


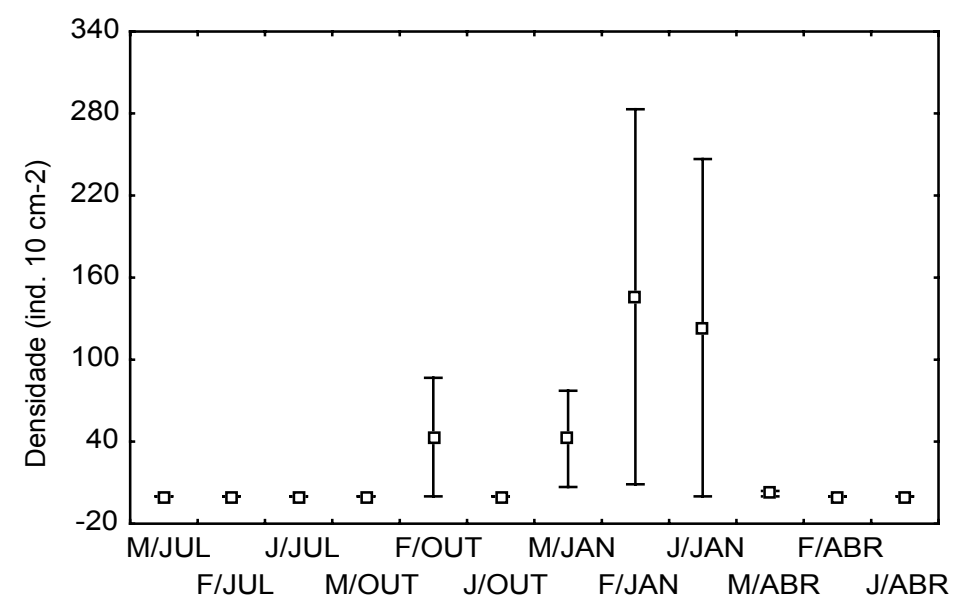

Figura 8 - Variação da densidade média e erro padrão dos Tardigrada no infralitoral durante a preamar na Ilha de Itamaracá, PE ( $\mathrm{M}=$ machos; F=fêmeas; $\mathrm{J}=$ jovens; Jul=julho, Out=outubro, Jan=janeiro, Abr=abril).

\section{DISCUSSÃO}

B. pennaki, única espécie de Tardigrada integrante da comunidade meiofaunística para a ilha de Itamaracá foi também apontada como a única espécie ocorrente no litoral sul do Pernambuco: baía de Tamandaré (Araújo e FonsêcaGenevois, 1988), baía de Suape ( Ayres e Fonsêca- Genevois, 1993), Restinga do Paiva (Victor Castro, 1998); e para o litoral central, no Istmo de Olinda ( Bezerra, 1994) o que indica, por um lado uma larga distribuição horizontal no Estado, sendo generalista para tipo de sedimento e para ambiente. Santos (1999) demonstrou não haver relação entre o tipo de sedimento e tipo de ambiente com a distribuição espacial de grupos da meiofauna no litoral brasileiro.

A variação de densidade observada entre os Tardigrada na área estudada, de 0 a 1300 ind. $10 \mathrm{~cm}^{-2}$, mostrou-se comparativamente maior que aquela registrada para praias não-estuarinas de zonas temperadas (De Zio e Grimaldi, 1966; Renaud-Debyser, 1963), e inferior à referida por Medeiros (1989) em praia arenosa do estado de São Paulo. Em região tropical típica a variação de densidade, assim como os picos, são sempre inferiores àquela aqui demonstrada. Esteves (1995), considera os Tardigrada um grupo raro e de ocorrência ocasional na Coroa do Avião (Itamaracá - PE - Brasil), localidade geograficamente próxima à estação 1 do presente trabalho, enquanto Pinto (1998) refere, para o mesmo local, a dominância de 10\% dos Tardigrada em relação à meiofauna total durante a época de estiagem, e $21 \%$ na época das chuvas. Ainda na Coroa do Avião foi registrada uma densidade de 180.000 ind. $10 \mathrm{~cm}^{-2}$ em amostras coletadas no início da baixa-mar (Fonsêca- Genevois et al.,1992). Almeida (1996) e Gomes (1999), trabalhando em ambiente estuarino do Canal de Santa Cruz, também próximo à região abordada no presente estudo, referem-se aos Tardigrada como sendo um táxon de ocorrência insignificante $(0.4 \%$ de abundância).Em praia arenosa da baía 42 
de Tamandaré, litoral sul do Estado, Silva (1997) cita um máximo de 70 ind.10 $\mathrm{cm}^{-2} \mathrm{e}$ Souza (1997), estudando a mesma praia encontrou o máximo de 418 ind.10 $\mathrm{cm}^{-2}$. Nascimento (1998) não menciona a presença do grupo em 14 estações localizadas nas praias urbanas do litoral pernambucano, cujas prospecções ocorreram durante época de estiagem. Vale ressaltar que, salvo Souza ( op.cit.) os demais autores citados trabalharam em regime de marés baixas, não investigando a influência do ciclo de marés sobre a distribuição da espécie. A autora, ao contrário do que aqui se atesta, concluiu que o efeito mareal não foi constatado para $92 \%$ dos grupos meiofaunísticos, mas que houve migração via água de enchente entre os Nematoda, Copepoda Harpacticoida e Turbellaria.

A densidade da meiofauna, em zonas litorâneas não-estuarinas, apresenta-se de 5 a 6 vezes menor que aquela de estuários, e 2 a 3 vezes maior que aquela de mar profundo (Renaud-Mornant et. al., 1984). Este fato é devido, entre outros fatores, à capacidade de reprodução de certas espécies: a rapidez de reprodução e maturação dos ovos é favorecida pelas particularidades físico-químicas do ambiente estuarino, o que possibilita, inclusive, a coexistência de indivíduos de duas faixas estárias bem distintas em uma mesma população. No presente estudo, B. pennaki apresentou dominância de jovens em duas épocas distintas do ano, indicando a ocorrência de duas fases reprodutivas na mesma população. Resta salientar que, tendo em vista o tamanho desses organismos ( menor que 100 micrômetros), seja ainda provável que a colonização de jovens na comunidade ocorra como efeito de deposição de partículas sedimentológicas sobre a zona intermareal. Tal processo é descrito por Gouleau (1975), demonstrando que a deposição lateral é mais eficiente na parte superior do terreno, existindo uma relação inversa entre tamanho de partícula e altimetria (as mais grosseiras depositadas em direção ao mar).

Foi detectado um ponto de intersecção para os padrões ecológicos das comunidades meiofaunísticas ocorrentes nas três estações estudadas: um possível confinamento desse táxon a trechos específicos da zona entre-marés, nos moldes daquele detectado em estuários por Guelorget e Perthuisot (1983), Gourbault e RenaudMornant (1986) e Fonsêca-Genevois (1987). Na área abordada pelo presente estudo, os Tardigrada dominaram numericamente a meiofauna em muitas ocasiões, no médiolitoral superior.

A dominância numérica de $B$. pennaki, principalmente na estação 1, em geral, ocorreu por ocasião das marés altas, sugerindo a existência de movimentos de dispersão impostos pela variação da maré, contrariando a assertiva de Fleeger e Decho (1987), que argumentaram serem estes animais maus colonizadores da coluna d'água, muito embora Renaud-Debyser (1963) cite as correntes de marés como um dos fatores ecológicos que condicionam a presença dos Tardigrada nos vários ambientes marinhos. Outros autores, como Palmer e Brandt (1981) e Palmer e Gust (1985), reconheçam a existência deste fator como importante mecanismo de dispersão para outros meiobentontes, como os Copepoda Harpacticoida.

\section{CONCLUSÕES}

Os Tardigrada na margem Sul da ilha de Itamaracá são representados unicamente pela espécie Batillipes pennaki Marcus, 1946, cuja distribuição mostra-se 
influenciada pelo regime de marés. No sentido espacial (três andares bênticos) o pico máximo de densidade da espécie foi registrado para o médio-litoral superior, distribuindo-se até o médio-litoral inferior ( valores inferiores de densidade) e esporadicamente até o infralitoral. É provável que os indivíduos se dispersem passivamente (por adesão aos grãos), sendo os processos hidrodinâmicos responsáveis pela dispersão e conseqüente colonização no meio intersticial. Tendo em vista que as populações de Tardigrada do litoral Sul de Pernambuco (baía de Tamandaré, especialmente) se compõem de $B$. pennaki, é aconselhável que se investigue a dispersão tanto no sentido vertical ( já que em ambientes estuarinos a camada oxiredutora é estreita ), quanto no sentido horizontal, seguindo este último a angulação da deriva litorânea. No sentido temporal a dominância de jovens em épocas distintas do ano (janeiro, pico máximo e julho, pico secundário) poderia indicar a coexistência de duas populações com períodos reprodutivos distintos, porém, pode-se questionar ainda se os jovens, por serem mais leves e de menor tamanho, não sigam o padrão de deposição de particulas sedimentológicas finas, mais eficiente para o pontos mais elevados do terreno. Foi justamente para o médio-litoral superior que se definiu um possível confinamento da espécie.

\section{AGRADECIMENTO}

Agradecemos à Dra. Jeanne Renaud-Mornant, do Museu Nacional de História Natural de Paris, por ter identificado a espécie de Tardigrada abordada neste trabalho.

\section{REFERÊNCIAS BIBLIOGRÁFICAS}

ARAÚJO, J. F. P.; FONSÊCA-GENEVOIS, V. Tardigrada do litoral de Pernambuco: Parte I. Zona intertidal da Baía de Tamandaré. In: Congr. Bras. De Zool., 15, 1988, Curitiba. Resumos...Curitiba, UFPR, 1988, p. 622.

AYRES, C. F. J. ; FONSÊCA-GENEVOIS, V. Estabilidade meiofaunística da Baía de Tamandaré: Prospecção em uma estação controle para o biomonitoramento litorâneo. In: Congr. Nord. de Ecologia, 5, 1993, Natal, Anais... Natal: UFRN, 1993, p. 103-104.

BEZERRA, T. N. C. Distribuição espaço-temporária da meiofauna do istmo de Olinda- PE, com especial referência aos Nematoda livres. Recife, 1994. 106 p. Dissertação (Mestrado). UFPE.

DE ZIO, S. ; GRIMALDI , P. Richerche sulla distribuzione ed ecologia di Batillipes pennaki Marcus, 1946. Uma spiaggia pugliese (Heterotardigrada).Cah. Biol. Mar. n. 5 , p. 271-285. 1964.

ELMGREN, R. Methods of sampling sublittoral soft bottom meiofauna. Oikos, n. 15, p. $112-120.1966$.

ESTEVES, A. M. Microdistribuição espacial da meiofauna na Coroa do Avião, Pernambuco. Recife, 1995, 74 p. Dissertação (Mestrado). Univ. Fed. de Pernambuco.

FLEEGER, J. W.; DECHO, A. Spatial variability of interstitial meiofauna: a review. Stygologia, v. 3, p. 35-54, 1987.

FONSÊCA - GENEVOIS, V. Ecologie des méio-et-mixofaunes d une vasière de $\mathbf{l}$ estuaire de la Loire. Correlations avec le millieu sedimentaire et ses eaux 
; INTERAMINENSE, L. J. L.; MENDES, A C. R. Meiofauna como estoque alimentício para aves limícolas na Coroa do Avião, litoral Norte de Pernambuco. In: Encontro de Zoologia do Nordeste, 9, 1992, Recife. Resumos... Recife: Soc. Nord. de Zool., 1992, 155 p., p. 42.

GOMES, C. A. A. Zonação da meiofauna no médiolitoral no manguesal de Itapissuma - PE , com ênfase aos Copepoda Harpacticoida. Recife, 1999, 36 p. Monografia (Graduação). Univ. Fed. de Pernambuco.

GOULEAU, D. T. Les premiers stades de la sédimentation sur le vasiéres littorales atlantiques. Rôle de l'emersion. Nantes, 1975. 245 p. +123 p. em anexo. Thése (Doctorat ès Sciences) - Université de Nantes.

GOURBAULT,N.; RENAUD-MORNANT, J.Le méiobenthos de la Ranse Maritime et la estructure des peuplements de Nématodes. Cah. Biol. Mar., v. 236, p. 409430, 1986.

GRIMALDI DE ZIO, S.; MORONE DE LUCIA, R. M.; D ADDABO GALLO,M.Marine tardigrades ecology. Oebalia, n. 9, p.15-31. 1983.

GUELORGET, O.;PERTHUISOT,J. P.Le domaine paralique.Expressions géologiques, biologiques et économiques du confinement. Travaux Lab. de Géologie. E. N. S., Paris, v. 16, 136p. 1983.

HIGGINS, R.P. International Symposium of Tardigrada. Mem. Ist. Ital. Idrobiol., n. 32, p. 14-470. 1974.

HULLINGS, N. C.; GRAY, J. S. A manual for the study of meiofauna. Smithsonian Contributions to Zoology. Smithsonian Institution Press. Washington, 84p. 1971.

MARCUS, E. Batillipes pennaki, a new marine Tardigrada from the North and South American Coast. Comun. Zool. Mus. Hist. Nat. Montev., 2 (33): 1-3. 1946.

MEDEIROS, L. R. Meiofauna de praia arenosa da Ilha Anchieta, São Paulo. São Paulo, 1989, 376 p. Dissertação (Mestrado). Univ. de São Paulo. Inst. de Biociências.

NASCIMENTO, A . P. Meiofauna mediolitorânea das praias urbanas de Pernambuco no período de seca. Recife, 1998, 68 p. Dissertação (Mestrado). UFPE.

PINTO, T. K. O. Estrutura da comunidade de meiofauna no banco de areia da Coroa do Avião, Itamaracá - PE - Brasil. Recife, 1998, 51 p.Dissertação (Mestrado).Univ. Fed. de Pernambuco.

PALMER, M.A.;BRANDT,R. R.Tidal variation in sediment densities of marine benthic Copepods. Mar. Ecol. Prog. Ser., 4: 207-212. 1981.

PALMER, M.;GUST, G. Dispersal of meiofauna in a turbulent tidal creek.J. Mar. Res.,43:179-210. 1985.

RENAUD-DEBYSER, J. Récherches ecologiques sur la faune interstitielle des sables. Bassin d Arcachon, Île de Bimini, Bahamas. Vie et millieu, n. 15, p. 1-157. 1963. 
SALVAT, B. Elements de properité des biotopes des sediments meubles intertidaux et écologie de leurs populations en microfaune et macrofaune. Vie et millieu, n. 14, p. 463-550. 1963.

RENAUD-MORNANT, J.; POLLOCK. L. W. A review of the systematics and ecology of marine Tardigrada. Smithson. Contr. Zool., 76: 109-117. 1971.

; BODIN, P.; BODIOU, J. Y.; BOUCHER, G.; BOVÉE, F.; CASTEL, J.; COINEAU, N.; TOURNIE, T.Estimations du rôle énergetique et dynamique spatio-temporelle du méiobenthos en millieu littoral: échantillonage et méthodologie. ATP - CNRS, 982002, Rapport Final, 232 p. 1981.

RÜTZLER, K. The mangrove community: aspects of its structure, faunistics and ecology. In: SIMPÓSIO INTERNACIONAL SOBRE LAGUNAS COSTEIRAS (Origem, dinâmica e produtividade). México. Lagunas costeras: Un Simposio. p. 515-536. 1967.

SANTOS, P. J. P. O meiobentos da costa brasileira: padrões de diversidade, de densidade e de dominância. In: Encontro de Zoologia do Nordeste, 12, 1999. Soc. Nordest. de Zool., 1999, p. $91-100$.

SILVA, G. S. Prospecção do meiobentos mediolitorâneo da baía de Tamandaré, litoral Sul de Pernambuco, com especial ênfase aos Acari. Recife,1997,110 p.Dissertação (Mestrado). Univ. Fed. de Pernambuco.

VICTOR - CASTRO, F. J. Impacto dos processos morfodinâmicos sobre a meiofauna da Restinga do Paiva - PE, Brasil. Recife, 1998, 70 p. Dissertação (Mestrado). UFPE. 